\title{
Pratiques de la culture des fruits
}

De l'ontologie appliquée au goût à Penang et au-delà

\section{Leo Mariani}

\section{(2) OpenEdition}

\section{Journals}

Édition électronique

URL : http://journals.openedition.org/ethnoecologie/3748

DOI : $10.4000 /$ ethnoecologie.3748

ISSN : 2267-2419

Éditeur

Laboratoire Eco-anthropologie et Ethnobiologie

Référence électronique

Leo Mariani, «Pratiques de la culture des fruits », Revue d'ethnoécologie [En ligne], 14 | 2018, mis en ligne le 31 décembre 2018, consulté le 02 mai 2019. URL : http://journals.openedition.org/ ethnoecologie/3748; DOI : 10.4000/ethnoecologie.3748

Ce document a été généré automatiquement le 2 mai 2019.

\section{cc) (†)}

Revue d'ethnoécologie est mis à disposition selon les termes de la licence Creative Commons Attribution - Pas d'Utilisation Commerciale - Pas de Modification 4.0 International. 


\title{
Pratiques de la culture des fruits
}

\author{
De l'ontologie appliquée au goût à Penang et au-delà
}

\author{
Leo Mariani
}

«Une question reste pour moi sans réponse : si
c'était les autres êtres vivants qui avaient éduqué
les hommes, si les chevaux leur avaient appris à
courir, les grenouilles à nager, les plantes à
patienter? ( André-Georges Haudricourt)
(Haudricourt \& Dibie $1987: 169)$

On envisage spontanément le goût comme la représentation subjective d'une sensation sapide. Une littérature récente a montré que cette interprétation est partiale, et que l'étude des façons de goûter et d'entrer en relation avec de la nourriture reste ouverte (Mintz 1996, Hennion 2007, Van de Port \& Mol 2015). On s'arrêtera notamment sur le fait que, dans toute l'Asie, le goût peut être associé à l'absence de saveur : appétence pour « la fadeur " (Jullien 1993) dans certains cas ou, comme on le verra dans ces lignes, attrait pour le numb taste. Ces goûts ont en commun de ne pas être sapides et d'interroger les catégories intuitives. Ils ouvrent aussi sur un versant mal connu de la sensibilité, où le goût se joue pour ainsi dire virtuellement, dans la disponibilité du dégustateur envers un ensemble d'expériences possibles plutôt qu'actuelles (Jullien 1993), et dans la capacité des aliments à soutenir et suggérer ces potentialités.

Contrairement à la fadeur, le numb taste ne laisse pas les papilles indifférentes: il engourdit la langue et l'intérieur des lèvres. C'est l'évocation d'un fruit d'Asie du Sud-est (le durian) qui permettra ici de discuter cette sensation rare. Shingo Hamada, un collègue japonais, m'a dit que des amateurs de fugu, poisson au venin mortel qui est l'objet d'un véritable culte culinaire au Japon, mentionnaient aussi le numb taste, perceptible quand l'animal est consommé très frais. En dehors du cas de certains durians, le seul autre exemple que j'ai personnellement éprouvé concerne Xanthoxylum piperitum, une petite baie que l'on appelle souvent " poivre du Sichuan ", en référence à la région de Chine où elle est traditionnellement cultivée. Dans ce cas, qui a l'avantage d'avoir été sérieusement documenté, on sait que la sensation d'engourdissement est induite par une molécule chimique : le sanshool (hydroxy- $\alpha$-sanshool). Mais, contrairement à ce que l'utilisation du 
terme "poivre" laisse penser, il faut distinguer les effets du sanshool de ceux qu'induisent la plupart des poivres. Comme les piments, ceux-ci contiennent en effet de la capsaïcine, une molécule qui est irritante en ce qu'elle active les récepteurs de chaleur des muqueuses de la bouche et de la langue. En bref, elle brûle. Le sanshool a un effet paresthésique: il induit un trouble mécanique de la sensibilité tactile en faisant littéralement vibrer certains récepteurs de la bouche à une fréquence que le cerveau ne peut pas informer (Hagura et al. 2013). D'un point de vue tactile (puisque c'est essentiellement de cela dont il s'agit), le numb taste se manifeste par une sensation subtile de pétillement, qui anesthésie légèrement les muqueuses de la bouche. Cette sensation peut durer plusieurs minutes et brouille la perception des autres saveurs. Pour ceux qui n'en ont pas l'habitude, elle n'est le plus souvent ni particulièrement agréable, ni vraiment désagréable, occasionnant des réactions amusées et embarrassées.

Figure 1 : Deux durians ouverts (l'un à chair jaune, l'autre à chair blanche)

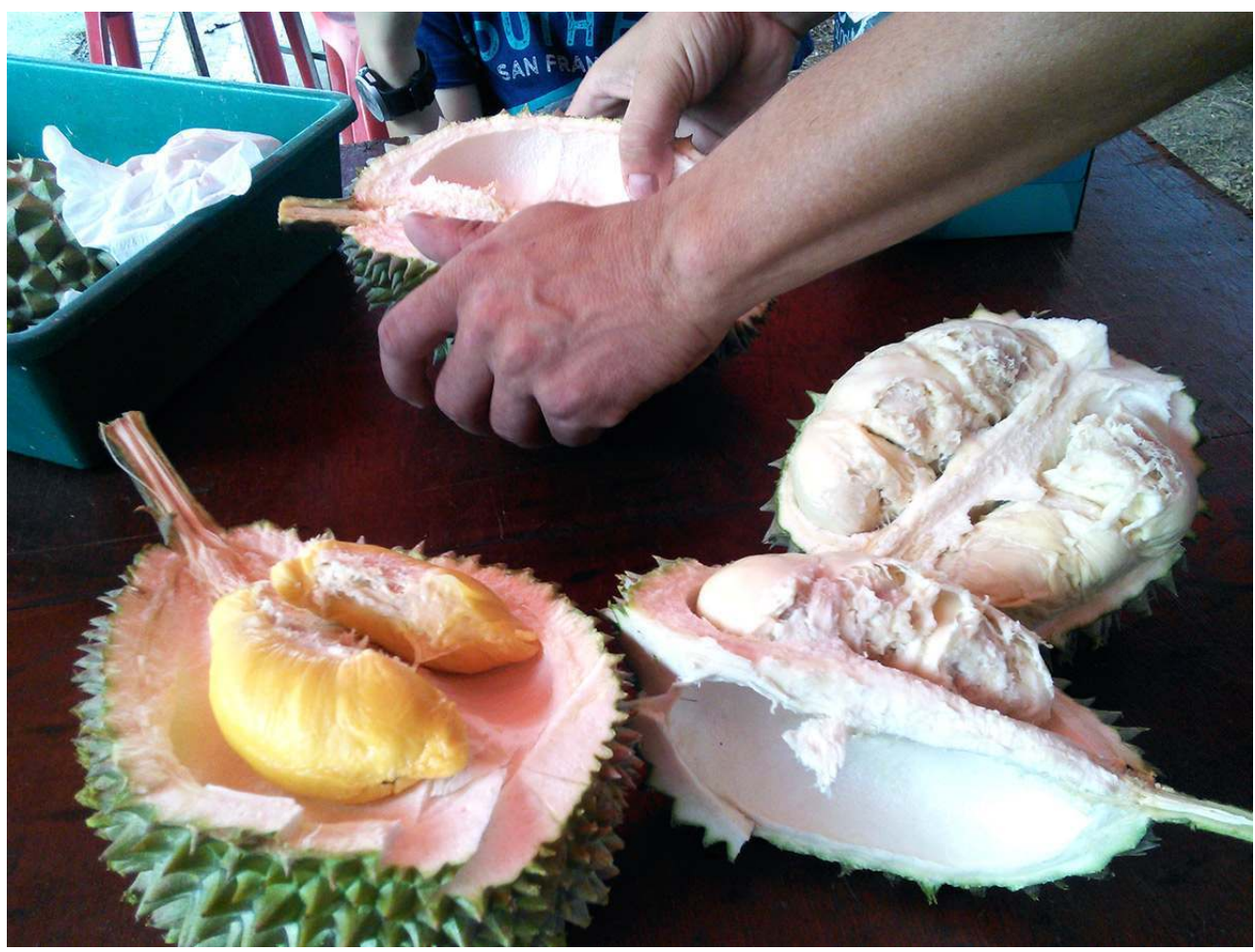

Au second plan, les mains sont en train de séparer deux pans d'une coque pour délivrer la chair qu'elles enferment

(c) L. Mariani - Penang (Georgetown), 2018

3 Les durians peuvent susciter le numb taste dans certaines conditions, de fraîcheur en particulier. Dans les plantations de Malaisie ${ }^{1}$, éprouver cette sensation d'engourdissement est relativement simple. Pour des raisons qui seront discutées, c'est moins évident à Singapour, au sud de la Péninsule, où beaucoup des meilleurs durians malaisiens sont exportés. Là, j'ai rencontré des amateurs qui évoquaient le numb taste avec piété, comme une expérience qu'il faut faire une fois dans sa vie. Pour certains, c'est donc une opportunité rare et ultime. Sinon, l'intérêt qu'elle suscite reste limité, même dans cette région, où la passion pour le durian est pourtant immense et les opportunités nombreuses. Il reste que le numb taste a trouvé l'un de ses plus ardents défenseurs sur l'île de Penang. Dans la suite, il sera nommé Tim, moins pour préserver son anonymat que pour ne pas parasiter son $e$-réputation. Tim est en effet producteur de durians, l'un des 
plus réputés de Penang, de Malaisie voire du monde. Il doit sa popularité à la qualité de ses fruits mais aussi à son discours esthète, notamment à sa promotion du numb taste, un goût dont il dit qu'il «contient tous les autres goûts» et qu'il a mis au cœur de l'expérience qu'il propose à ses clients.

Le numb taste va servir ici d'entrée privilégiée vers la question plus générale des rapports humains aux aliments, qui sera abordée dans sa transversalité, en liant la connaissance, la consommation et la production. Dans sa théorie de l'esthétique, Luigi Pareyson $(2007: 21)$ ${ }^{2}$ proposait d'envisager les formes (entendues comme expressions sensibles des propriétés d'un matériau) : « dans l'acte de conclure, et en même temps d'inclure, le mouvement de production qui [les] oriente et trouve en elle[s] son propre succès ». Appliquée "aux formes durians ", cette idée doit permettre de penser la façon dont les mondes humains sont vécus en même temps qu'ils sont composés. Faire ces liens implique que l'on se garde de toute inflation théorique pour se concentrer sur les jeux de relations que l'ethnographie du numb taste et de son contexte permettent de mettre au jour. Il faudra ensuite conceptualiser ces rapports, extraire et discuter leurs caractéristiques communes.

Dans la plupart des mythes d'origines connus, ni le langage ni la culture ne sont posés comme éléments de différentiation entre humains et animaux, mais la domestication du feu qui sert à cuire les aliments (Lévi-Strauss 1964). Les rapports aux aliments sont donc au cœur de la définition que les humains se donnent d'eux-mêmes. Dans le même temps, ils constituent l'un des grands enjeux écologiques de demain. Si l'avenir de la planète est lié à la biodiversité, alors on conclura qu'il se joue dans la définition de l'humanité, plus particulièrement dans l'organisation des rapports existentiels que les hommes nouent avec le monde.

Il convient, dans une première partie, de décrire le contexte général de la production et de la consommation de durians en Malaisie et à Singapour, contexte mal connu qui se caractérise par une contrainte agronomique pour le moins contradictoire avec les principes de l'agriculture moderne. La deuxième partie s'attache à la description et à l'analyse des pratiques de Tim, et de son goût pour numb taste, que l'on replacera dans la production malaisienne globale en le conceptualisant comme un type de disposition envers le végétal. La dernière partie viendra comme une synthèse, s'attachant d'une part à lier l'engagement gustatif de Tim dans le monde à des pratiques économiques et agricoles, et d'autre part à remettre le cas dans une perspective régionale et globale. Il s'agira in fine de réfléchir aux conséquences pratiques et sensibles de choix ontologiques. 
Figure 2 : Un étal de durians en train d'être achalandé

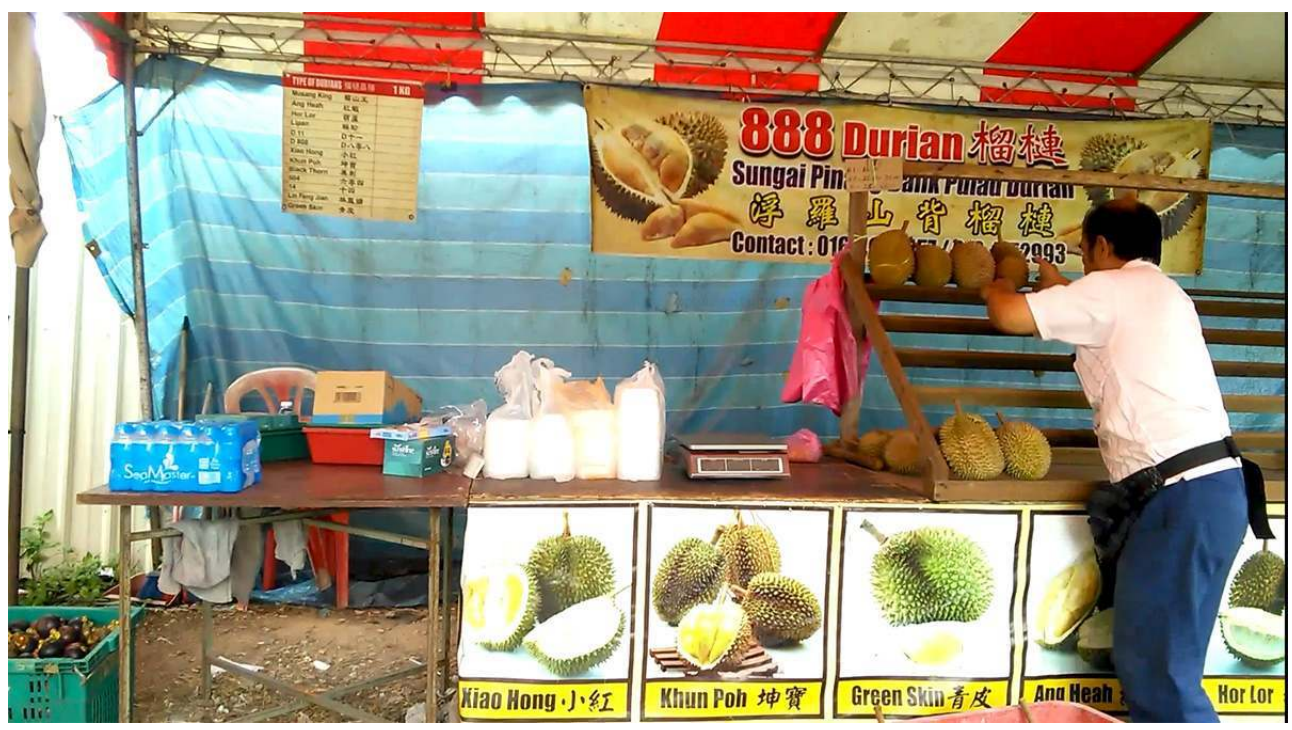

Les fruits sont en général classés par variétés (on perçoit un tableau des prix en haut à gauche) et consommés en arrière, sur des tables. Beaucoup de vendeurs (et de producteurs) de durians sont d'origine chinoise, ce qui explique les idéogrammes. En bas à gauche de la photo, on notera enfin la présence d'une caisse de mangoustans, qui sont souvent consommés après les durians, pour rafraîchir le corps (le durian étant considéré comme un fruit très chaud, en médecine chinoise mais aussi dans les savoirs populaires)

(c) L. Mariani - Penang (Georgetown), 2018

\section{De l'attente et d'un principe ontologique qui change tout}

7 Il semblera curieux, à certains lecteurs, que l'odeur du durian n'ait pas encore été mentionnée. Le fruit a pourtant la réputation de sentir très mauvais ; «la mort », « les excréments » ou «le vomi » si l'on se fie aux centaines de témoignages livrés dans la littérature de voyage et aux rumeurs qui courent sur Internet. Cette focalisation sur l'odeur du durian est en réalité le produit d'une histoire singulière, qui mêle rapports coloniaux, définition des corps et molécules chimiques, une histoire qui est d'abord celle des populations occidentales (Mariani 2015, 2017 et 2018). L'odeur sera quoi qu'il en soit absente des lignes qui suivent, pour la simple raison qu'elle n'a même jamais été évoquée sur les terrains mobilisés dans cet article.

8 L'île de Penang, située à quelques encablures de la côte occidentale malaisienne, est un haut-lieu de production de durians. Des gens viennent du monde entier pour visiter les plantations qui émaillent les pentes escarpées de son versant ouest, où se côtoient des arbres de tous âges et de toutes sortes, du plus sauvage au mieux domestiqué. C'est en effet l'une des particularités de l'endroit que de montrer encore une grande diversité de cultigènes et de modes de culture du fruit. Sur un petit périmètre, on trouve aussi bien des durians sans pedigree (kampong durians, «durians de village »), ramassés en forêt ou élevés à l'arrière d'une maison, qu'une impressionnante collection de cultivars issus de plantations professionnelles, la réputation de certains d'entre eux dépassant de loin les frontières de l'île ou du pays ${ }^{3}$ (Figure 3). Alors que dans toute la Malaisie la production tend plutôt à s'uniformiser, en se modernisant et en se resserrant autour de quelques 
variétés de durians à fort potentiel économique ${ }^{4}$, Penang reste pour l'heure un parangon de diversité. L'île est exiguë, les durians l'habitent depuis longtemps et la forêt est difficile à contenir. Elle se mêle aux cultures et aux jardins, facilitant les pollinisations croisées, les repousses spontanées, le bouturage et la greffe, et donc l'émergence de nouveaux fruits. Certains durians sont vendus au bord des routes par des particuliers pour quelques centimes d'euros, d'autres sont l'objet d'un commerce officiel et peuvent coûter cent fois plus cher (jusqu'à vingt euros le kilo) ${ }^{5}$. De manière générale, les prix dépendent du volume de la production et donc de l'avancée de la saison, des variétés de fruits, de leur calibre, de leur fraîcheur et de leur provenance, car des gens payent cher pour goûter les durians de producteurs renommés, voire de quelques arbres particuliers. Les tarifs peuvent également varier d'un jour à l'autre, en fonction des conditions climatiques: par exemple les pluies qui tombent alors que les durians sont mûrs, modifient la texture de leur chair (qui se gorge d'eau) et diluent leurs arômes. De même, les vents violents, qui induisent la chute et la mise sur le marché de spécimens manquant de maturité, font baisser les cours.

Figure 3 : Dans un magasin de durians et de produits locaux ouvert par l'État malaisien à Singapour

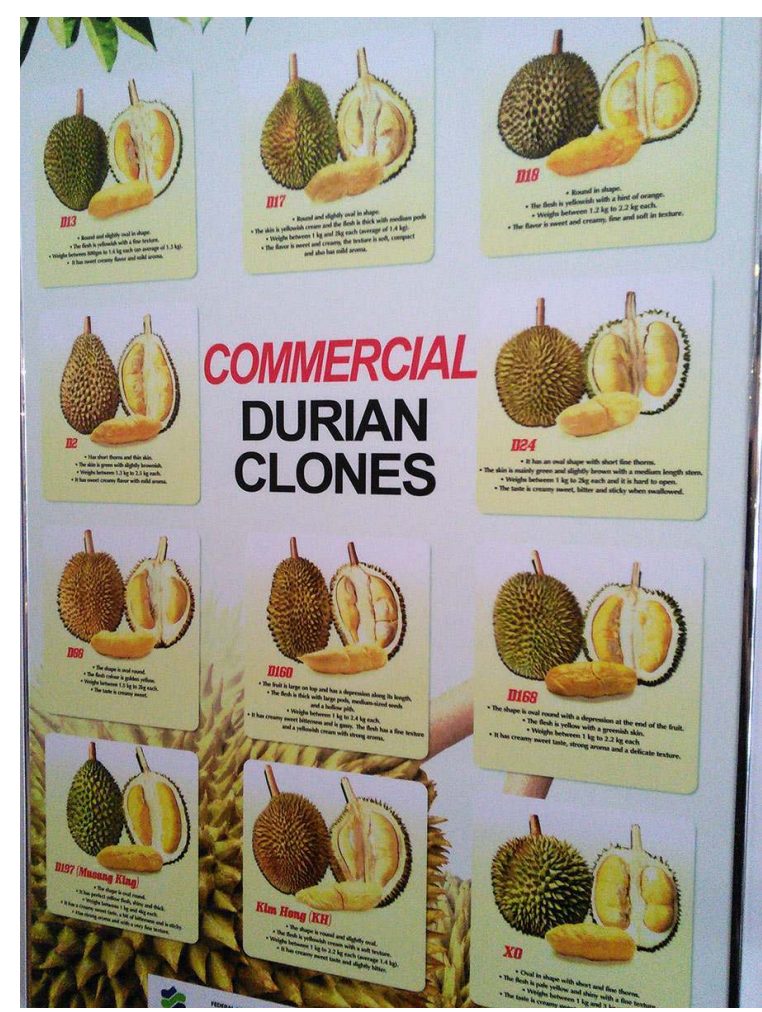

Une affiche qui présente brièvement les caractéristiques physiques et les profils aromatiques de quelques-uns des cultigènes les plus populaires produits dans le pays

(c) L. Mariani - Singapour, 2015

Contrairement à ce qu'on observe dans d'autres régions de Malaisie, les meilleurs spécimens produits à Penang ne sont pas tous exportés, peut-être parce que le trajet est long jusqu'à Singapour (où les durians malaisiens de catégorie supérieure sont majoritairement consommés) et que le processus de sénescence du fruit est rapide (voir plus loin). Mais l'isolement relatif de la production locale par rapport au débouché naturel que constitue la petite cité État ne s'explique pas seulement par des contraintes. Si Penang exporte en effet peu de durians, elle est en revanche le théâtre d'un important 
tourisme dédié au fruit, depuis Singapour justement, mais aussi depuis les zones urbaines de Malaisie, le sud de la Chine, Taiwan ou Hong Kong (et, depuis peu, en provenance de pays occidentaux). Ce tourisme gastronomique, qui forme le principal débouché pour la production locale haut de gamme existe ailleurs dans le pays, mais nulle part il n'atteint un tel niveau. En plus de la qualité globale des fruits produits, il faut dire qu'il a sans doute été stimulé par «la marque Penang ", sur laquelle il était facile de capitaliser (l'île est connue pour ses plages et sa capitale, Georgetown, ancien comptoir de la compagnie anglaise des Indes orientales, est aujourd'hui classée au patrimoine mondial de l'UNESCO). Il est probable, en outre, que le tourisme gastronomique se soit appuyé sur le développement récent de compagnies aériennes régionales à bas coûts, qui relient l'aéroport international de Georgetown à toute l'Asie; et sur l'aisance avec laquelle certains producteurs locaux se sont appropriés l'usage des réseaux sociaux, où ils sont soutenus par une communauté passionnée et enthousiaste.

10 En Malaisie (et à Singapour), c'est l'attente qui caractérise en premier lieu l'expérience d'un amateur de durians. Elle est d'abord liée à la saisonnalité du fruit, qui est respectée dans tout le pays ${ }^{6}$, et qui le rend d'autant plus désirable qu'elle est limitée. Partout, en Malaisie et à Singapour, le durian est considéré, sans équivoque, comme «le roi des fruits", le meilleur de tous. L'ouverture de la saison ne passe donc inaperçue pour personne. Pour nombre d'habitants de ces pays, elle constitue même un événement de première importance (Figure 4).

Figure 4 : Des touristes se prennent en photo devant une représentation du durian « roi des fruits ", dans le hall d'entrée de l'aéroport international de Singapour

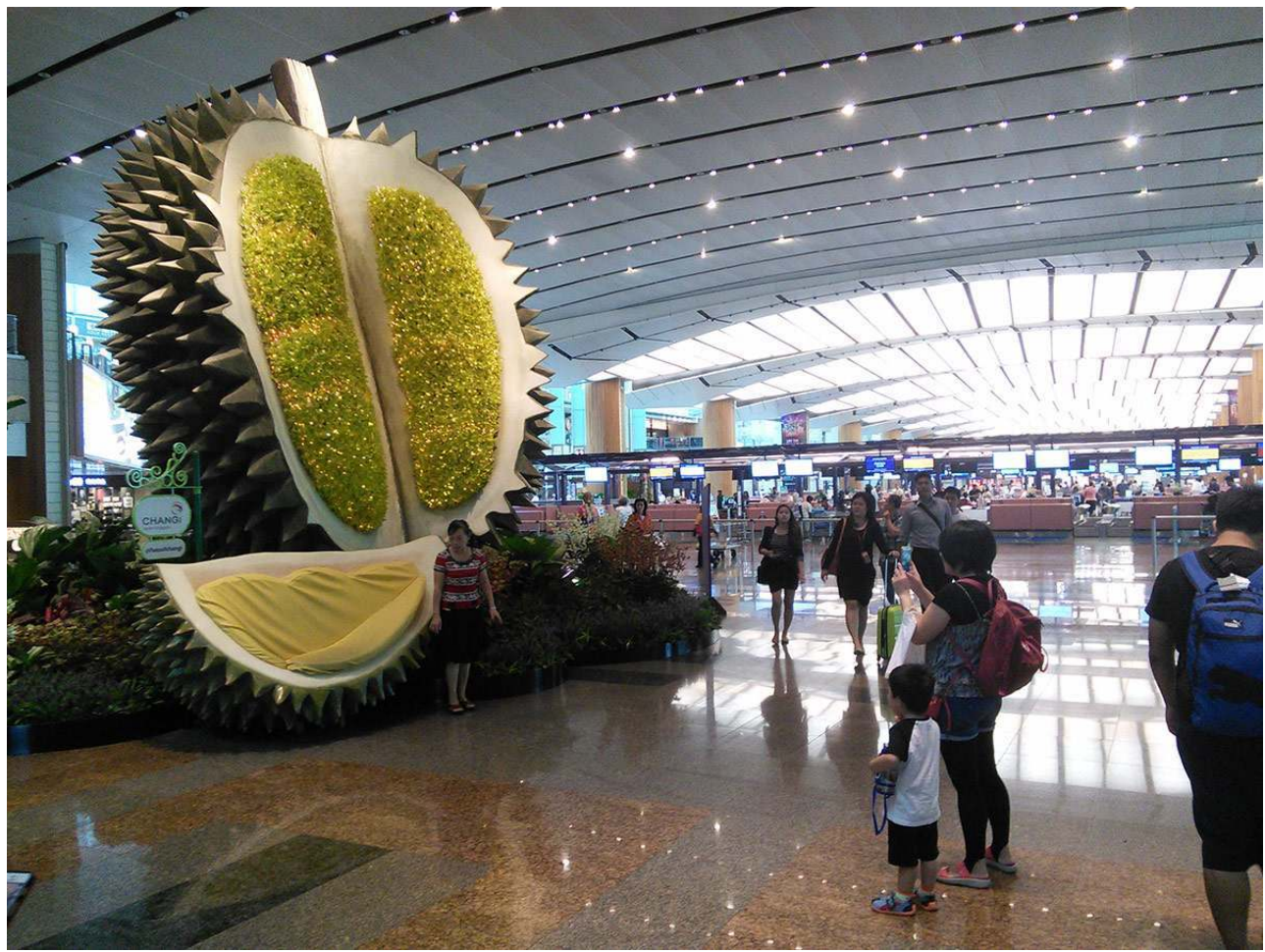

(C) L. Mariani - Singapour, 2015

11 Mais l'attente est aussi conditionnée par un autre impératif qui, pendant la saison ellemême, détermine fondamentalement la consommation, la production et le commerce. Cet impératif veut que jamais les durians ne soient prélevés sur l'arbre, car ils doivent tomber 
d'eux-mêmes. Il faut s'arrêter sur ce point, qui est déterminant dans la compréhension du marché régional et dans l'analyse développée ici. On ne peut imaginer, en effet, défi plus radical aux fondamentaux de l'agriculture et du commerce modernes que ce principe qui freine la rationalisation de la production et de la consommation, en même temps qu'il réduit dramatiquement le temps de conservation des durians. En Malaisie pourtant (et à Singapour), le marché lui est entièrement subordonné.

Certains des témoignages que j'ai recueillis justifient cette astreinte comme une réponse à la nécessité de respecter «le caractère » de l'arbre, à qui des intentions sont volontiers prêtées, ainsi qu'une forme d'éthique ${ }^{7}$ et la tendance à punir les impatients qui ponctionneraient ses fruits avant terme en ne produisant plus l'année suivante. Cette préséance accordée à l'arbre a des conséquences pratiques effectives. Elle doit donc être interprétée comme une disposition fondamentale (ontologique) du rapport humains/ végétal, où le second est assimilé à un partenaire avec lequel il faut composer plutôt qu'à un objet qui servirait passivement des desseins humains. Il est entendu, d'ailleurs, que le durian a, le cas échéant, les moyens de se défendre: en ne donnant plus de fruits. La plupart des Malaisiens (et des Singapouriens) perçoivent très bien ce que cette orientation a de singulier, et ils aiment l'invoquer lorsqu'ils parlent d'eux-mêmes ou qu'ils cherchent à se définir (voir par exemple Yoon Wah 2013). Ainsi, les longs aprèsmidis passés dans les plantations à surveiller la chute des durians constituent-ils, dans le pays, un motif récurrent de la littérature, des arts et des discours sur la «Malaisianité ». De même, la préséance accordée au végétal est fréquemment mise en avant lorsque référence est faite à la Thaïlande voisine, car si ce pays s'enorgueillit lui aussi de sa production de durians, les fruits y sont en revanche toujours récoltés avant terme. Ces prélèvements, qui sont associés à une culture annualisée et à un niveau d'industrialisation globalement bien supérieur, caractérisent un mode de production au sein duquel, contrairement au cas malaisien, l'humain dispose d'une priorité sur le végétal ${ }^{8}$. De façon tout à fait intéressante, ce mode de production dominé par l'emprise humaine est associé à " un goût " pour les durians qui contraste lui aussi avec " le goût »" malaisien, puisque les Thaïlandais préfèrent les fruits qui ne sont pas encore matures (alors qu'ils pourraient attendre qu'ils mûrissent pour les consommer). Ainsi, et même si l'appétence pour les fruits verts est très répandue en Thaillande, il est tentant de l'associer, dans le cas du durian, avec les pratiques techniques d'une agriculture qui anticipe sa maturité. Le rapprochement est d'autant plus séduisant que, symétriquement, il fonctionne très bien du côté Malaisien où, parallèlement aux considérations sur le statut de l'arbre, les gens revendiquent simplement leur désir de consommer les durians à maturité parfaite. D'un côté, donc, le durian est apprécié quand il n'est pas à terme, et il se trouve qu'il est effectivement prélevé avant terme. De l'autre c'est quand il est mûr qu'il est le meilleurs, et on le laisse opportunément tomber. Le goût pour les fruits coïncide autrement dit avec des pratiques (techniques) agricoles et, dans un cas au moins, avec un postulat ontologique portant sur la façon de hiérarchiser le vivant. Même si l'on ne peut pas, dans ces lignes, privilégier un ordre de causalité en identifiant qui, du goût, des pratiques ou du principe d'organisation préexiste (qui, parmi ces éléments « contraint » vraiment les autres ?), l'association est tout sauf anodine. Quoiqu'elle soit encore grossière pour le moment, gardons-la en mémoire pour la suite.

13 Enfin, il faut insister sur la remarquable diffusion de ces différentes pratiques. Tout visiteur pourra ainsi facilement vérifier que, pour les Malaisiens et les Singapouriens, les durians doivent tomber d'eux-mêmes... et qu'ils le font effectivement. Sur les étals de 
Thaillande, il aura en revanche toutes les peines du monde à trouver un seul durian qui n'a pas été prélevé sur l'arbre. Pour l'heure, l'astreinte que les premiers s'imposent n'est pas négociable ${ }^{10}$, tout du moins lorsque la production locale est en cause. En revanche, chacun sait que les durians thaïs qui sont vendus pour des prix modiques tout au long de l'année ont fini leur maturation loin de leur arbre d'origine. On ajoutera que même les voleurs de fruits (assez nombreux lorsqu'il s'agit de durians), qui chapardent sur les terres d'autrui, respectent cet impératif. À Singapour, où la production de durians est aussi maigre que la passion qu'il suscite est dévorante, des gens peuvent ainsi passer des nuits entières dans les forêts domaniales à espérer la chute de fruits qu'il leur serait infiniment plus facile de détacher manuellement. En Malaisie dans les plantations, des employés sont chargés de surveiller les arbres et de réunir les durians au fur et à mesure. Pour éviter qu'ils ne dévalent les pentes escarpées ou ne soient abîmés dans leur chute, des filets sont parfois tendus au-dessous des ramures (Figure 5). Sur certains arbres réputés pour la qualité de leur production, les fruits (on peut en compter une centaine) sont même attachés un par un à l'aide de petits cordages noués sur les troncs, à hauteur d'homme, et reliés aux pédoncules après avoir été passés au-dessus des branches porteuses. Lorsqu'un durian tombe, il est de cette façon maintenu en suspens jusqu'au moment où il sera descendu manuellement.

Figure 5 : Plaine située à l'ouest de Penang

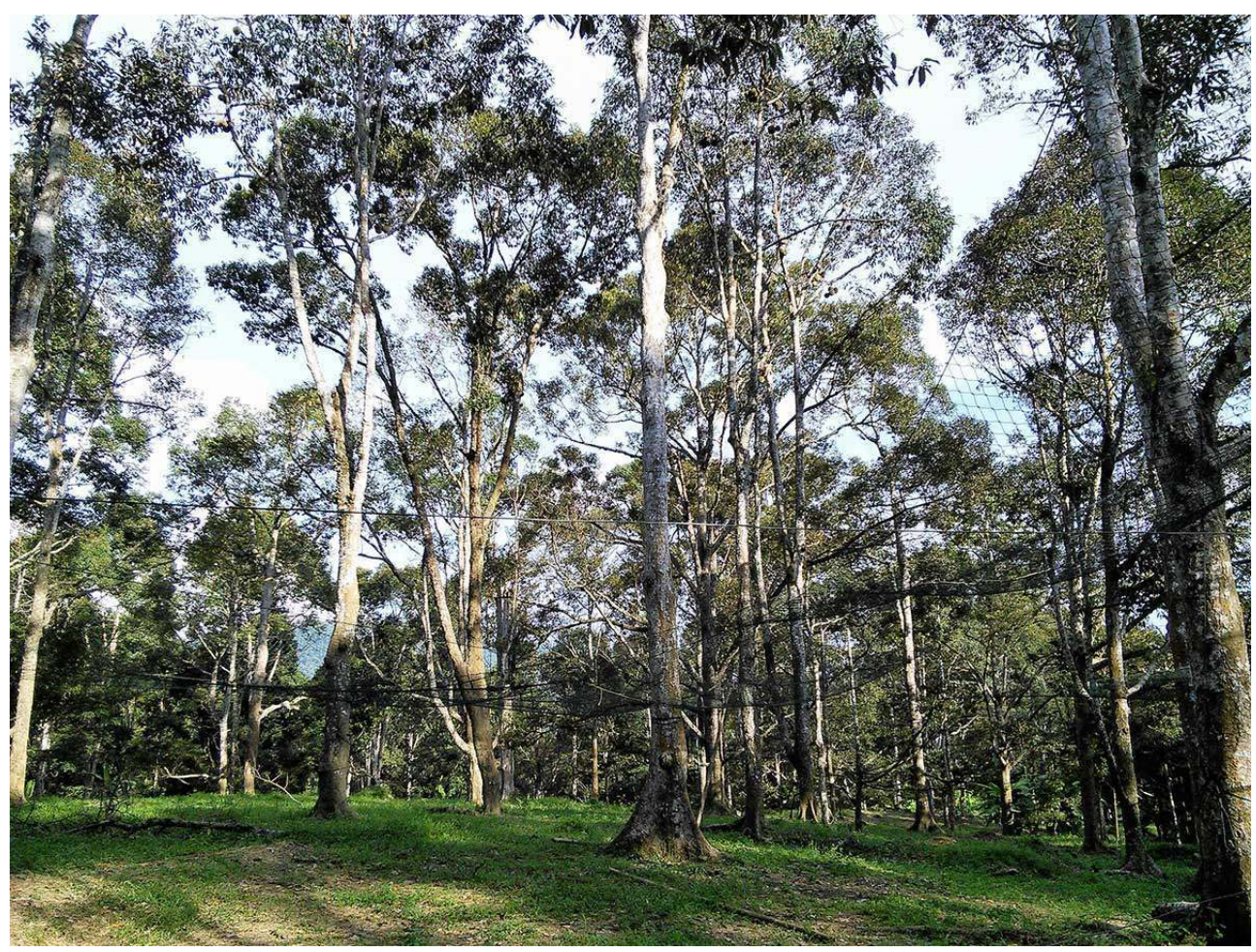

Des arbres probablement assez âgés (car de grande taille), et qui n'ont vraisemblablement pas été greffés (les arbres greffés sont plus petits, et ils vieillissent moins). On devine les filets tendus sous les ramures pour amortir la chute des fruits

(c) L. Mariani - Penang, 2014 


\section{Trouble du goût}

14 Tim reçoit ses clients sur une terrasse ombragée, entourée de plantations, où des employés amènent les durians au fur et à mesure de la journée. Souvent, les fruits sont transportés dans des paniers qui pèsent jusqu'à cent kilos, à l'arrière de petites motos dont les allées et venues durent de l'aube au crépuscule. Mais la majorité des durians arrive le matin, parce que les fruits tombent plutôt la nuit (Figures 6 et 7).

Figure 6 : De très hauts arbres (une trentaine de mètres) portant des fruits

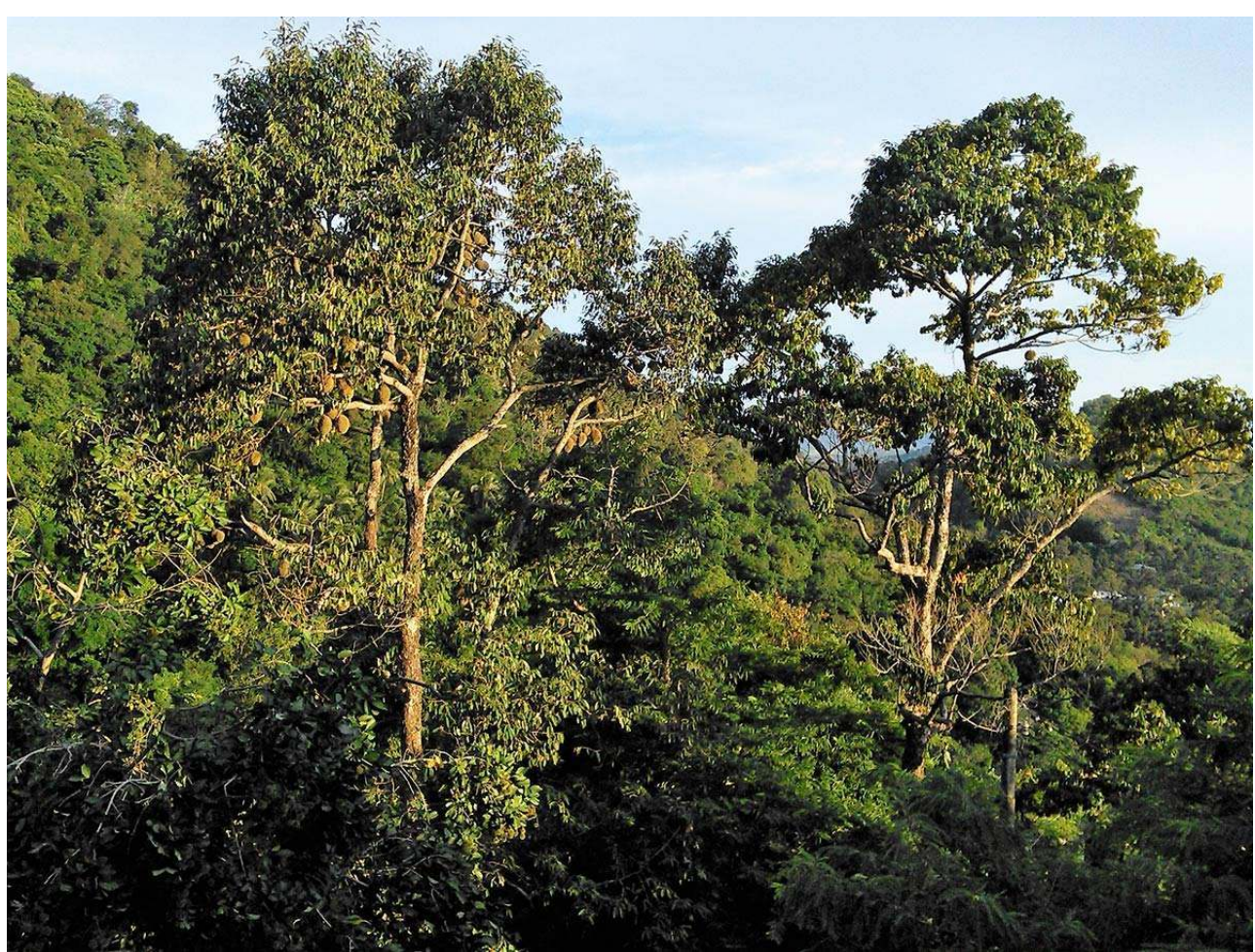

(c) L. Mariani - Penang, 2014 




(c) L. Mariani - Penang, 2014

Dans l'ensemble, le goût des amateurs malaisiens et singapouriens dépend beaucoup de la fraîcheur des durians. À Singapour et dans les grandes villes malaisiennes, ce sont les fruits de la dernière nuit qui sont de loin les plus prisés (ou ceux du matin quand ils arrivent l'après-midi). Les chances de ceux qui sont tombés la veille de trouver preneur s'amenuisent irrémédiablement, si bien que les fruits restés sur les étals finiront par être vendus à des tarifs en deçà des standards qui ont cours lors de l'arrivée des camions qui effectuent les rotations depuis les lieux de production ${ }^{11}$. Il semble que l'exigence de fraîcheur suive elle aussi une contrainte technique, car elle tend à augmenter avec la proximité des plantations. Dans le cas de Tim, elle est poussée à son paroxysme, au point que les fruits perdent quasiment tout intérêt après quelques heures (avant que les citadins n'y aient seulement accès) : « ils sont morts ».

Fixer « la mort du fruit » à un moment plutôt qu'à un autre relève assurément d'un choix partial, qui se fonde ici sur des critères chimiques et laisse deviner, en creux, les contours d'une conception pragmatique de "la vie comme mouvement", dans le sens que lui donnait William James (Latour 2004). C'est en effet durant les premières heures qui suivent la chute de l'arbre que le durian connaît normalement ses évolutions les plus spectaculaires. Dans cet intervalle, il est pris d'une succession rapides et perceptibles de modifications, qui contrastent avec l'état d'inertie (de stabilité) relative auquel il parvient ensuite, après que ses arômes se sont spécifiés ${ }^{12}$. D'après Tim, l'amorce du processus de sénescence est provoquée par la collision du fruit avec le sol. L'impact "réveille» le durian : c'est pourquoi les fruits qui sont tombés dans les filets placés sous les ramures sont frappés par terre, parce qu'ils sont encore "endormis ». À l'impact succède une période d'incertitudes, sur laquelle se concentre toute l'attention de Tim. On ignore alors 
précisément « où le durian va aller », s'il va plutôt développer l'amertume qui plaitt tant à certains et/ou la douceur qui sied à d'autres ${ }^{13}$. Dans certains cas, le doute et le trouble sont encore renforcés par l'apparition d'un goût tout à fait unique, qui gêne la plupart des gens mais dont Tim soutient qu'il est le plus digne d'intérêt, parce qu'il « contient tous les autres », en potentiel : « le numb taste ». Pour l'heure ce goût ne semble pas avoir retenu l'attention des chimistes, et l'on devra se contenter d'expliquer sa survenue par une hypothèse. Un spécialiste de la chimie des fruits m'a ainsi suggéré que son apparition pourrait être associée à la production d'éthylène de stress, consécutive à la collision. Cette hormone, qui est à l'origine de la maturation des fruits, a un impact sur la production de nombreux autres composés chimiques, et sa grande volatilité justifierait que le phénomène soit limité dans le temps ${ }^{14}$. Toujours est-il que l'effet paresthésique induit est rare, et que dans la pratique il reste imprévisible. Si les fruits issus de vieux arbres et de certaines variétés ont, selon Tim, plus de chances de susciter le numb taste, rien ne le garantit non plus.

17 Tous les matins de la saison, Tim attend donc qu'on lui amène les durians tombés pendant la nuit, sans qu'il puisse exactement savoir à quand remonte leur chute. Avant de les proposer à la dégustation, il va donc devoir les regrouper et les ordonner en fonction de leur niveau d'évolution et de leur potentiel. Parce que les durians ne sont jamais ouverts avant d'être mangés ${ }^{15}$, l'expertise à laquelle ils sont soumis est réalisée sans contact direct avec la chair, qui est abritée sous une épaisse enveloppe épineuse. S'en suit une évaluation au cas par cas, réalisée avec l'aisance et la vélocité de l'habitude, mais qui mobilise un répertoire complexe d'indices dans lequel l'homme puise au besoin. Ainsi les fruits vont être tour à tour, et selon les cas :

- examinés visuellement (leur forme, l'écartement de leurs épines, leur couleur ou l'apparence de la sève qui suinte des pédoncules permettent de se faire une idée de l'âge de l'arbre d'origine et de la fraîcheur du fruit - toutes ces informations sont pondérées en fonction de la variété quand elle peut être déterminée) ;

- soupesés (ce qui permet d'estimer le volume des graines et donc d'avoir des indications sur la densité de la chair);

- sentis en intercalant le nez entre le pouce et l'index posés sur la coque (ce qui permet d'obtenir des informations générales sur le potentiel du fruit, et de percevoir le numb taste - Tim sent les durians dans leur partie haute, car ils y mûrissent moins rapidement, ce qui, selon lui, donne des indications plus fiables sur la maturité de l'ensemble);

- secoués légèrement (le durian est un fruit à arille, si bien que lorsqu'on le remue d'un geste sec celles-ci se déplacent dans leur compartiment - la spontanéité et la facilité de ces déplacements apportent des indications sur la maturité et sur la qualité des arilles, qui doivent bouger, car cela signifie qu'elles commencent à se détacher des parois et donc à " confire ", mais pas trop, car un mouvement trop perceptible signe une maturité excessive);

- tapotés avec les ongles, un bâton ou le revers d'un couteau (le son que le durian produit est aussi un bon indicateur de la maturité - mat il suggère qu'il est trop tôt, creux qu'il est temps).

L'expertise dont les durians font l'objet n'est pas l'affaire d'un seul instant, car Tim doit en permanence adapter la temporalité des fruits à celle de ses visiteurs, garder un œil sur l'évolution des premiers, ajuster leur répartition en la corrélant aux arrivées de clients et de nouveaux durians. Ainsi se met en scène une chorégraphie ininterrompue, tout entière organisée autour des fruits. Les changements dont ils sont le siège sont au cœur de 
l'expérience que Tim propose à ses visiteurs, qui sont invités à suivre les évolutions d'un durian d'abord « endormi », ou bien à oublier un autre déjà ouvert sur le coin de la table, avant d'y revenir plus tard. Tout est ici question d'adaptation à l'incertitude, et de valorisation de la contingence. Autrement dit, Tim mène une pluralité de dialogues avec des durians individualisés, des fruits qui, chacun leur tour, sont pris dans un rapport sensible et attentif qui est sans cesse remis en jeu. Dans cette relation toujours-en-trainde-se-faire, l'attention du producteur est tendue vers une qualité qui est elle aussi instable : le numb taste, ce goût « qui contient tous les goûts possibles» et qui se déploie dans un moment de pur potentiel. L'expérience gustative est ici dans ce que le durian suggère, une forme d'au-delà de lui-même qui, cela mérite d'être souligné, illustre un principe esthétique dont Sabban (1996) et Jullien (1993) relèvent la récurrence dans l'histoire de la Chine et de ses populations.

Il reste que le numb taste déstabilise la plupart de ceux qui le découvrent, et les clients de Tim ne font pas exception, eux qui trouvent habituellement leur bonheur dans les saveurs bien individuées et les plaisirs moins équivoques qu'offrent les fruits (relativement) stabilisés que l'on trouve sur la plupart des étals de Malaisie et de Singapour, ou même ceux de Tim lorsqu'ils ne sont plus troublés par le numb taste... Ces fruits qui, pour le producteur, « sont morts parce qu'ils ne bougent plus ». Le goût de Tim n'est pas un goût photographique, il n'évoque pas un tableau que l'on pourrait s'attacher à décrire ${ }^{16}$. C'est un goût-événement ou, si l'on me permet le jeu de mot, un goût du grouillement. Le goût du calme avant la tempête, sensation évanescente ou intuition que l'on peut avoir de capter, un instant, l'énergie potentielle de tous les devenirs. Jamais ce goût ne s'actualise de façon sapide. Il se réalise, en amont de toute forme de détermination, dans la rencontre momentanée d'un goûteur et d'un goûté ouverts sur un éventail virtuel. Cet instant n'est pas donné, il doit être recherché. En ce sens, Tim est «à la manière du chasseur primitif », il « habite » le goût, car il s'oriente « le long d'un trajet plutôt que sur un plan » (Leroi-Gourhan cité par Ingold (2013: 26)). Du chasseur primitif Tim a l'instinct du pisteur, qui rejette sa proie lorsque le fil de la traque est rompu par la prise. En pratique, il goûte d'ailleurs à peine les fruits, ou bien du bout des lèvres, comme s'il lui suffisait de capter ce moment d'épure où le goût n'est pas encore goût, où l'appétit reste ouvert, aiguisé plutôt que consommé.

En somme, Tim ne se contente pas de faire avec la contingence des fruits (ce qui est déjà beaucoup) : il la valorise en mettant leur autonomie, leur variabilité et leur diversité au cœur de l'expérience proposée à ses clients. Derrière le numb taste, ce sont des fruits « vivants » qu'il montre à ses visiteurs et sur la piste desquels il guide leur attention, avec une approche tout en retrait et en précautions. Finalement, Tim ne prend l'initiative du rapport qu'au moment où il frappe les durians encore « endormis » pour les réveiller. On ne s'étonnera pas, en conséquence, qu'il ne se qualifie jamais lui-même de "producteur " de durians, car cela reviendrait à s'attribuer la paternité des fruits, mais plutôt "d'accoucheur", comme pour marquer sa position extérieure et ouverte «d'accueillant».

\section{Contingences et universalité. Le temps des fruits, ou celui des humains}

21 Le cas de Tim n'est pas représentatif, mais il a l'avantage de mettre en relief les fondements du rapport humains/durians que l'on trouve à travers toute la Malaisie et 
Singapour. Ce rapport, on a vu qu'il était foncièrement contingent, dans la mesure où il est conditionné par la chute du fruit: il serait plus exact de dire « de chaque fruit » car, dans cette configuration, chaque durian nécessite un moment d'attention. Cette chute, les humains peuvent éventuellement en estimer la survenue, mais ils ne peuvent pas la prévoir. Ils ne peuvent pas non plus savoir exactement quel volume de fruits sera obtenu le lendemain, ni de quelle variété ou de quel arbre ils seront issus. En laissant l'initiative au durian, les producteurs malaisiens vont à l'encontre de la rationalité économique. Ils compliquent la gestion des stocks et ils réduisent aussi très considérablement les débouchés pour leur production, parce que le durian qui s'est décroché de lui-même est beaucoup plus fragile et périssable que celui qui a été prélevé dix jours avant maturité. Il devient alors plus risqué de le faire voyager, parce qu'il s'abîme vite et qu'on s'est beaucoup rapproché du moment fatidique où il sera gâté. Cela explique sûrement que les durians malaisiens ne soient pas exportés au-delà de Singapour et que, dans cette partie de l'Asie du Sud-est, la chaîne formée par les humains et les durians ne connaisse pas de temps mort: du ramassage à la consommation, toutes les étapes sont réalisées en flux tendu. En tombant, le durian amorce une course contre la montre qui emporte tout sur son passage. Les fruits doivent être réunis, triés, puis transportés au plus vite sur les lieux de vente. Aux premières heures du jour, les commerçants de Singapour sont en contact avec leurs fournisseurs. Ils estiment l'heure d'arrivée des camions, prennent connaissance du détail des cargaisons, et des tarifs. Déjà ces informations sont relayées sur les réseaux sociaux, les téléphones sonnent, les réservations pleuvent et les travailleurs singapouriens organisent leurs pauses ou leur passage après le travail. Durant les quelques mois de la saison, il en va chaque jour ainsi : le durian impose ses conditions. Pour les meilleurs fruits, moins de 12 heures s'écouleront entre la chute de l'arbre (parfois à 8 heures de route de Singapour) et le moment de la consommation. Plus tard, les durians de qualité inférieure seront soldés, certains seront transformés, parfois cuits, mais c'est en général le dernier recours, pour ne pas gaspiller.

Le rapport de Tim avec ses fruits offre une illustration condensée de la chaîne qui vient d'être décrite, du point de vue de sa temporalité et de sa spatialisation en particulier. Pour partie, cette différence de paramétrage doit être attribuée au producteur, parce que les durians ne "vivent» selon lui que quelques heures, autour du numb taste. Cette comestibilité très éphémère a pour effet d'exacerber leur influence sur l'organisation de l'ensemble et sur sa spatialisation, parce qu'elle les rive littéralement au lieu de leur production. En principe (dans l'absolu), rien n'empêche en effet ces durians d'être consommés ailleurs. Il suffirait qu'ils soient transportés à Singapour avec beaucoup d'autres. Mais il ne s'agirait plus alors que de fruits parmi d'autres, pas de « ces durians là », qui se définissent avec Tim et chez lui. « Ces durians-là » n'existent vraiment que là, portés par les contingences inextricables qui les font tels qu'ils sont, et qui dans le même temps organisent l'expérience que l'on peut faire d'eux ${ }^{17}$. Dans cet enchevêtrement, qu'il serait plus approprié de qualifier de concrescence, Tim est un passeur ou un montreur, mais c'est le fruit qui subordonne l'ensemble. C'est lui qui entraîne l'inventivité des hommes dans sa chute, lui qui les force à s'adapter. En dernier ressort, c'est aussi lui qui pousse les curieux à parcourir des milliers de kilomètres pour faire l'expérience de ce qu'il ne peut offrir que dans les conditions précises où il se trouve. En attribuant plus haut tout le mérite du développement du tourisme gastronomique à Penang au discours des hommes, à leur ingéniosité et à leurs stratégies de valorisation, on s'était quelque peu laissé rattraper par un réflexe anthropo-centré assez commun. Il serait plus équitable, 
maintenant, de rendre au fruit ce qui lui appartient et que les Malaisiens ne cherchent pas à lui ôter : le privilège de l'initiative. C'est, au passage, tout le commerce du durian sur l'île qui pourrait être relu comme une adaptation (une valorisation) à la priorité donnée à l'arbre dans les relations. On ne contestera pas, évidemment, que les conditions humaines et environnementales de Penang étaient favorables au développement d'un tourisme dédié. Mais les touristes n'ont pas eu non plus d'autre choix que d'aller vers des durians qui, de toute façon, ne pouvaient concrètement pas venir à eux. Ce sont ces fruits, valorisés pour les liens singuliers qu'ils portaient et continuent de porter, qui sont sans doute les meilleurs avocats de la production insulaire. Ce sont eux qui font rêver les gourmets de Singapour (pourtant bien fournis), et qui les incitent à prendre l'avion pour un weekend.

La priorité donnée à l'arbre dans toute la Malaisie cadre l'ensemble des pratiques observables dans le pays. Les exemples qui ont été présentés et discutés montrent qu'elle induit une forme de tropisme diffus, « une pente » ou une courbure que la temporalité du fruit impose aux mondes humains. Dans les plantations de Tim comme sur les étals de Singapour, c'est le temps du fruit qui conditionne tout, depuis les pratiques techniques et spatiales des uns et des autres jusqu'à leurs sociabilités et leurs horaires de travail. Bien sûr, il y a différents niveaux de tension dans cet espace, dont la cambrure s'intensifie à mesure que l'on approche des lieux de production, à la manière de ces représentations de l'espace-temps par les théories de la relativité. On saisira ainsi l'occasion pour situer Tim là où la déformation du plan est la plus profonde, à proximité d'une étoile (d'un durian). On placera l'île de Penang autour, puis les réseaux qui courent jusqu'à Singapour au-delà. Pour l'heure, l'espace-temps des fruits malaisiens ne s'étend pas plus, parce que les moyens disponibles ne le permettent pas. Il reste tout entier tourné vers les lieux de production (et vers les fruits), orienté de façon centripète ${ }^{18}$. Seule la réputation des fruits fait écho par delà les frontières, au point que, comme les autres, le Sultan de Brunei prend parfois son avion (personnel) pour venir se servir en durians frais à Singapour (alors qu'il habite une île où l'on produit aussi beaucoup de durians).

Il faut revenir, enfin, sur l'opposition avec le cas thaillandais, que les Malaisiens et les Singapouriens pointent lorsqu'ils veulent appuyer sur ce qui les singularise. Il existe, en Thaillande comme ailleurs, différents types d'usages et de pratiques du durian, mais tous ont en commun de sectionner les fruits avant maturité. Au moment zéro de la relation, leur temporalité est donc subordonnée à celle des humains, et c'est une propriété qui influence forcément l'ensemble des rapports possibles. On ne cherchera pas à statuer ici sur les motivations profondes de ce geste, mais on évoquera ce qu'il permet, et ce qu'il empêche. Certains des avantages induits sont évidents, notamment ceux qui découlent de l'apparition de stocks : «terrain de jeux [...] pour le traitement de masse » (Guille-Escuret 2018: 91). Dès lors que les fruits ne marquent plus eux-mêmes le moment de leur consommation, c'est en effet aux humains qu'il appartient de tout décider. Ils peuvent alors choisir de prélever les durians en amont, puis de les conserver dans des chambres froides par exemple. Les fruits deviennent alors exportables au bout du monde ${ }^{19}$, et même en Malaisie ou à Singapour, où ils sont moins onéreux qu'une production locale qu'ils remplacent du reste pendant huit mois de l'année. Couper les durians c'est aussi, et de fait, la condition sine qua non du développement d'une agriculture qui chercherait à rationaliser ses procédures et ses coûts, comme le fait très bien l'agriculture industrielle. Beaucoup d'exploitants (le terme prend tout son sens ici) thaïlandais ne s'y sont pas trompés et ils ont développé une culture annualisée, qui épuise les arbres mais qui 
produit des fruits en quantité (parce que les taux de mortalité sylvicole peuvent être eux aussi planifiés). Aujourd'hui, cette culture s'organise essentiellement autour de la production de trois variétés à gros rendement (alors que plus de 300 sont officiellement enregistrées dans le pays), dont le comportement agronomique est contrôlé d'un bout à l'autre de la chaîne, cadré par des procédures rationalisées qui décomptent les temps de production en jours et qui mécanisent la pollinisation, le traitement et le ramassage (Figure 8). C'est là une agriculture humaine, seulement humaine, qui fixe et qui subordonne le comportement agronomique des végétaux (Bonneuil \& Thomas 2010) à des impératifs qui n'appartiennent qu'à elle. Cette agriculture a des avantages, parce qu'elle permet par exemple de garantir une qualité moyenne de fruits, et des revenus réguliers. En réduisant les effets de contingence, la dépendance des végétaux aux lieux et à ceux qui les cultivent, elle écrase toutefois leurs qualités (notamment leur temporalité) sous celles que les humains leur attribuent. Dans ces conditions, on conçoit que le goût de Tim ne puisse simplement pas advenir. On imagine aussi que la fixation et la standardisation des fruits d'un point de vue agronomique tend à induire corrélativement la fixation et la standardisation des goûts, puisque c'est précisément l'une des qualités des fruits produits que de garantir des qualités organoleptiques stables. On voit bien, ainsi, que le rapport existentiel exprimé par le goût se retrouve dupliqué dans la gestion des environnements.

Figure 8 : Durian suspendu à la branche qui le porte par son pédoncule

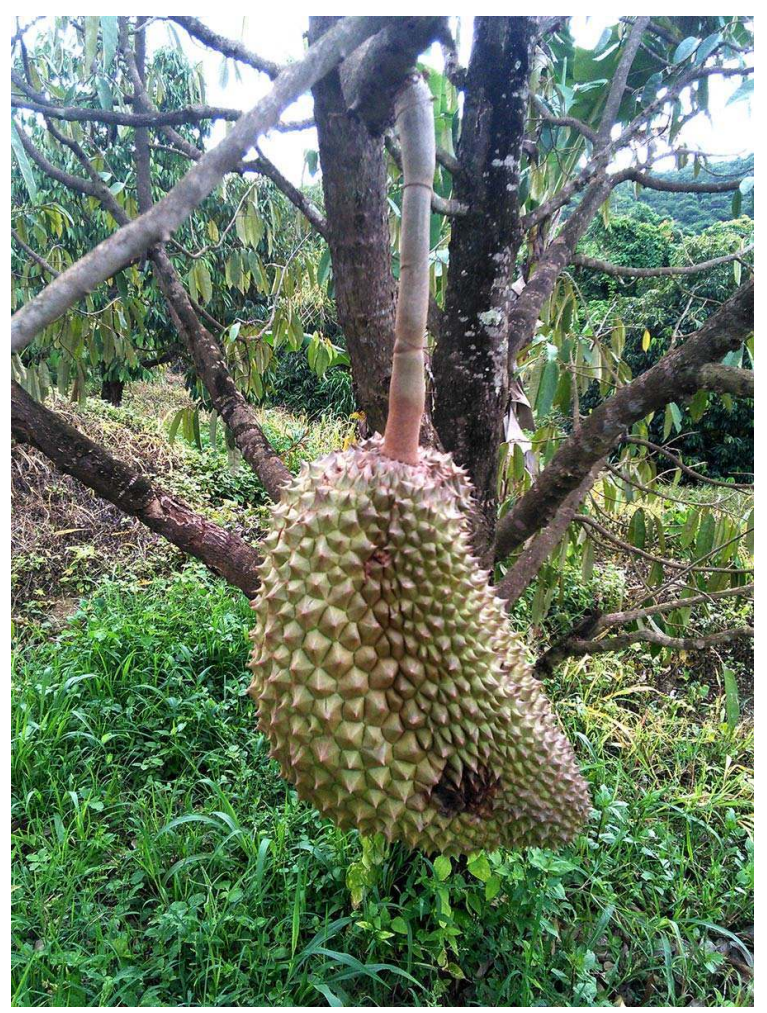

Le fruit a une forme inhabituelle. II est issu d'une variété thaï très commune et particulièrement productive (Monthong) que l'on trouve aujourd'hui en abondance en Thaïlande, au Cambodge et au Vietnam, ainsi qu'en Indonésie (mais pas en Malaisie)

(c) L. Mariani - Cambodge, 2014

La Thaillande et la Malaisie se situent de part et d'autre d'une ligne de crête. Sans doute y a-t-il, dans ces pays, des situations limites qui se ressemblent. Mais la pente est raide, de chaque côté. Donner la priorité à l'arbre ou à l'humain n'est pas innocent, cela ne libère 
pas les mêmes opportunités, agronomiques et existentielles. Beaucoup de projets, dans le monde, visent à valoriser la diversité de productions locales : les pommes de terre ou les maïs en Amérique du Sud, les fromages ici, les vins ailleurs. Leurs intentions sont sans doute bonnes. Pourtant, fixer la diversité (pour la préserver) pose problème, ou du moins question. Il est rare, en effet, que les groupes humains qui produisent de la diversité le fassent pour produire de la diversité. Les inciter à le faire pourrait bien les détourner de leurs préoccupations, autrement dit de la façon par laquelle ils définissent ce qui compte d'abord pour eux : la satisfaction d'un goût, de besoins, de sociabilités, d'idées ou même de leur curiosité. Peut-être, alors, que les réponses à la déflation de biodiversité qu'on observe partout se situent (aussi) ailleurs que dans la gestion explicite de la biodiversité. Est-ce, autrement dit et comme le soutenait le Général de Gaulle, parce qu'un pays comme la France produit tant de fromages différents qu'il est ingouvernable ? Ou bien, comme le demande Guille-Escuret (1988: 23), parce qu'il est impossible à gouverner qu'il a pu élargir la gamme de ses fromages? La question est d'une incontestable actualité. En Malaisie, les variétés de durians se créent, s'échangent et se diffusent encore beaucoup plus vite qu'elles ne se stabilisent et qu'elles ne se fixent (même si certaines variétés à succès tendent à dominer le marché). Elles sont associées à des dizaines de créations sauvages, spontanées, qui naissent des pollinisations croisées et de la dissémination "naturelle». Pour l'heure, aucun cadre légal ou même programmatique ne vient pourtant réglementer cette diversité, qui est générée par la collaboration intéressée mais ouverte des humains avec les arbres.

\section{Conclusion}

Quelques décennies en arrière, un auteur dont les travaux n'ont pas été traduits en anglais (Haudricourt 1962) a formalisé un ensemble d'oppositions auxquelles la présente étude fait écho. Distinguant d'abord un mode de traitement des végétaux qui procède par action indirecte négative d'un autre fondé sur l'action directe positive, il déclinait ensuite ces contrastes dans deux archétypes: un modèle "proche-oriental», caractérisé par l'utilisation des céréales, un contact direct, une attitude directive et une pression sélective (un contrôle accru sur la reproduction des végétaux); et un modèle " océanien ", où les hommes privilégient une relation indirecte avec les tubercules, une aide négative (protection par des préparations du sol autour des boutures) et une curiosité collectionneuse (accumulation de clones ayant des dispositions différentes). Il ajoutait que l'opposition de ces modes d'actions se déclinait dans toutes les formes de relations, en particulier dans les rapports entre humains. Je ne m'aventurerai pas, ici, sur ce dernier point. En revanche, on voit très bien les correspondances avec l'étude qui vient d'être menée et, pour élargir encore les comparaisons, on devrait ajouter que chacun de ces modes de productions oriente des perspectives existentielles (voir aussi Mariani 2018). La sélection et la stabilisation de variétés de fruits qui finissent par se présenter à tous et partout de la même façon ne fait pas qu'affecter le monde. Elle touche les consommateurs en retour, ne serait-ce que parce que, mieux assurés de trouver ce qu'ils attendent (et rarement déçus), ils ne sont plus forcés de renouveler leur engagement envers les aliments, de les re-sentir ou de les re-toucher pour les connaître. À l'opposé, les aliments contingents (dont les durians de Tim sont des parangons) sont des incitateurs. Ils forcent à chaque fois leur chance : il faut les tester pour savoir de quoi il ressort. D'un côté les fruits sont finalement l'expression fidèle d'une classe ou d'une 
catégorie que chacun d'eux incarne, et en cela ils sont abstraits, de l'autre ils constituent une perpétuelle remise en cause catégorielle, ils sont contingents. Les uns peuvent être facilement consommés pour ce qu'ils sont (ce que le travail de fixation réalisé en amont garantit), et à ce titre ils tendront à solliciter prioritairement la cognition. Les autres imposent que l'on éprouve ce qu'ils font, et pour cette raison ils engageront plus fondamentalement les sens.

La curiosité collectionneuse de Tim et sa passion pour les fruits ont l'avantage d'enrichir à la fois la biodiversité et la connaissance du monde vécu. En revanche elles coûtent cher, elles ne sont pas démocratiques et leur hyper-localisation ne va pas sans poser des problèmes écologiques (puisqu'il faut venir en faire l'expérience en avion). Les durians produits dans les plantations industrielles de Thaillande ne font l'économie d'aucun problème écologique, mais ils peuvent être goûtés par tous et partout. Ils ne créent pas de privilèges... Mais ne créent pas non plus d'expériences ou de connaissances originales du monde. Ils ne nous apprennent pas que le goût pour un curieux pétillement peut changer les existences et modifier la face d'un lieu et des réseaux qui lui sont associés. Les premiers, dont la valeur est contingente, exercent une force d'attraction parce qu'ils suscitent un désir rare, d'ailleurs souvent déçu, alors que les autres sont dans un mouvement centrifuge d'expansion qui les fait tendre vers plus d'universalité, i.e. vers la satisfaction d'un nombre toujours plus grand de désirs. On se souvient que le goût de Tim relevait plus de la mise en appétit que de la consommation. Curiosité pour les choses ou satisfaction de leurs désirs, compagnonnage interspécifique ou domestication, toujours les humains semblent hésiter à prendre toute la place, ou à s'accommoder avec les nonhumains. Ils ont en tous cas ce choix, dans lequel leur engagement corporel et leur humanité se définissent.

\section{BIBLIOGRAPHIE}

Bonneuil C. \& Thomas F. 2010 - Purifying landscapes: The Vichy Regime and the Genetic Modernization of France. Historical Studies in Natural Sciences 40 (4) : 532-568.

Guille-Escuret G. 1988 - La Souche, la cuve et la bouteille. Les rencontres de l'histoire et de la nature dans un aliment : le vin. Paris, Éditions de la Maison des Sciences de l'Homme, 197 p.

Guille-Escuret G. 2018 - Structures sociales et système naturels. L'assemblage scientifique est-il réalisable ?. Londres, ISTE editions, $233 \mathrm{p}$.

Hagura N., Barber H. \& Haggard P. 2013 - Food vibrations: Asian spice sets lips trembling.

Proceedings of the Royal Society B 280 (1770). [En ligne] URL : http://

rspb.royalsocietypublishing.org/content/280/1770/20131680 Consulté le 24 novembre 2018.

Haudricourt A.-G. 1962 - Domestication des animaux, culture des plantes et traitement d'autrui. L'Homme 2 (1) : 40-50.

Haudricourt A.-G. \& Dibie P. 1987 - Les pieds sur terre. Paris, A.M. Métailié, 197 p. 
Hennion A. 2007 - Those things that hold us together: taste and sociology. Cultural Sociology 1 : 97-114.

Ingold T. 2013 - Une Brève histoire des lignes. Trad. de l'anglais par Sophie Renaut. Bruxelles, Zones Sensibles, 256 p.

Jullien F. 1993 - Éloge de la fadeur. À partir de la pensée et de l'esthétique de la Chine. Paris, Le Livre de Poche, $158 \mathrm{p}$.

Latour B. 2004 - How to Talk About the Body? The Normative Dimension of Science Studies. Body \& Society $10(2-3): 205-29$.

Lévi-Strauss C. 1964 - Mythologiques, t.1: Le Cru et le cuit. Paris, Plon, 403 p.

Mariani L. 2015 - L'Exotisme et le fruit de l'imagination : le-durian-qui-partage-l'humanité-endeux. Anthropologie et Sociétés 39 (1-2) : 313-328.

Mariani L. 2017 - Une odeur d'enfer. À propos du devenir universel des qualités organoleptiques d'un fruit. Techniques \& Culture $62: 48-67$.

Mariani L. 2018 - Matières à manger. Propositions pour penser les rapports humains/aliments. Revue d'Anthropologie des Connaissances 12 (3) : 429-453.

Mintz S. 1996 - Tasting food, tasting Freedom: excursions into eating, culture and the past. Boston, Beacon, $149 \mathrm{p}$.

Pareyson L. 2007 - Esthétique: Théorie de la formativité. Paris, Édition Rue d'Ulm, 352 p. [En ligne] URL : https://books.openedition.org/editionsulm/959 Consulté le 24 novembre 2018.

Sabban F. 1996 - Art et culture contre science et technique. Les enjeux culturels et identitaires de la gastronomie chinoise face à l'Occident. L'Homme 36 (137) : 163-193.

Van de Port M. \& Mol A. 2015 - Chupar Frutas in Salvador da Bahia: a case of practice-specific alterities. Journal of the Royal Anthropological Institute 21 (1) : 165-180.

Yoon Wah W. 2013 - Durians Are Not the Only Fruit. Notes From the Tropics. Singapour, Epigram books, 175 p.

\section{NOTES}

1. Le travail présenté ici est le fruit de séjours réalisés entre 2014 et 2018, en Malaisie et à Singapour (pour un total de six mois). Notons que les deux pays seront souvent évoqués de concert. Du point de vue du durian et de son commerce, ils forment en effet un seul ensemble, ne serait-ce que parce que Singapour absorbe une bonne partie de la production malaisienne. Mais les deux pays ont de plus une longue histoire commune, n'étant indépendants l'un de l'autre que depuis 1965. Les compositions de leurs populations sont également comparables, même si les proportions ethniques qui les caractérisent divergent (héritages de la colonisation anglaise notamment, les populations singapouriennes et malaisiennes sont principalement composées de Chinois, d'Indiens et de Malais).

2. Je remercie Simon Gérard de m'avoir suggéré cette lecture.

3. Comme le vin a ses cépages à succès, le durian a ses variétés réputées. Certaines sont anciennes (les premières variétés malaisiennes officiellement enregistrées, D1 et D2, l'ont été en 1934), d'autres ont juste quelques années (les derniers enregistrements datent de 2017). Pour exemple, citons la variété la plus emblématique de Penang (plus ancienne que ne le laisse penser son enregistrement officiel, en 1990), appelée Red prawn (Udang merah en malais, Hang hae pour les locuteurs chinois), et une variété plus récente (2012) qui rencontre un grand succès : Black thorns 
(Duri hitam en malais, Ochee pour les locuteurs chinois). Notons qu'il existe plus de variétés de durians cultivées en Malaisie que les 200 entrées (environ) des registres du gouvernement ne le laissent penser. En effet, beaucoup «d'inventeurs» n'engagent pas les procédures d'enregistrement.

4. Ce resserrement est relatif, surtout comparé à ce qu'on observe ailleurs. Pour s'assurer une pollinisation efficace, une plantation de durian doit quoi qu'il en soit comporter au moins quatre ou cinq cultigènes différents. Si les fleurs sont hermaphrodites, pistils et étamines ne sont pas fertiles en même temps et il est plus prudent de miser sur une floraison étalée. Les fleurs sont principalement pollinisées par des chauves-souris, oiseaux et abeilles. Cependant, le succès de certaines variétés, comme celui du cultivar D197 (Musang King, Raja Kunyit ou Maosang wang) ces 20 dernières années, incite les producteurs à adapter leur production à la demande (en regreffant des arbres, car planter prend plus de temps).

5. Sachant que la chair constitue environ un tiers du poids total, on a une idée du prix très élevé que coûtent certains durians (les fruits malais font en moyenne 1,5 kilos).

6. La Malaisie continentale est soumise à deux régimes de mousson, ce qui est dû au positionnement du pays entre la mer d'Andaman et la mer de Chine, dont les températures sont différentes. On peut y diviser la production de durians en trois zones. À Penang, le pic de production a lieu entre juin et juillet. Un peu plus bas sur la côte occidentale, jusqu'à Singapour (où la production est symbolique), la saison est décalée d'un mois environ. Enfin, dans l'est du pays et dans les hautes terres du centre, elle démarre en juillet et finit en septembre. Il arrive, dans toutes les régions, que les arbres produisent une deuxième fois dans l'année, mais en quantités moindres, aux alentours de décembre, janvier. Grâce à un système d'importation continu depuis toutes ces régions, Singapour est sans doute l'endroit où l'on peut, le plus longtemps, manger des durians malaisiens.

7. L'arbre est censé ne pas laisser tomber ses fruits sur la tête des bonnes personnes, en particulier sur celles des enfants, qui traversent souvent les plantations, sur le chemin de l'école notamment.

8. On reviendra plus loin sur ce point, mais il est évident que cette particularité est infiniment plus cohérente avec les principes de la rationalité économique capitaliste.

9. Dans cette phrase, le double usage du singulier pour "goût » ne sous-entend pas qu'il n'y aurait qu'un seul goût dans un cas comme dans l'autre. Le terme évoque un principe (de maturité d'un côté, de verdeur de l'autre) qui, dans chacun des cas, est commun à tous les goûts.

10. On observe d'ailleurs que ce choix culturel conditionne jusqu'aux programmes de recherches mis en place par le gouvernement malaisien, qui incorporent tous cette nécessité : il s'agit de développer des moyens de conservation qui permettraient de mieux exporter les fruits après qu'ils soient tombés et en préservant leurs arômes (que la congélation et les chambres froides abîment).

11. Ou bien ils seront vendus à des néophytes, qui n'ont pas les moyens de discuter la qualité.

12. Le fruit ne cesse évidemment pas d'évoluer, mais il évolue beaucoup moins vite.

13. L'amertume, la douceur et le doux/amer constituent les principaux critères du choix d'un fruit sur les étals, à quoi il faut ajouter leur variété, certaines ayant la réputation de développer plutôt l'une ou l'autre de ces qualités générales. Les durians proposent infiniment plus d'arômes, mais les gens ne cherchent pas souvent à les qualifier.

14. Je remercie Michel Génard pour ces suggestions prudentes, dont l'utilisation n'engage que moi.

15. Il arrive que l'on voie, sur les étals de Malaisie et de Singapour, de la chair de durian empaquetée. Ce conditionnement est réservé aux fruits de moindre qualité, et destiné aux publics moins exigeants et/ou argentés. Notons que c'est là une autre différence de taille avec les pratiques thaillandaises car, dans le pays voisin, la chair des fruits est souvent pré-conditionnée, quelle que soit sa qualité. En Thailande, on observe aussi une pratique qui consiste à découper 
une lucarne dans la coque des fruits, dégageant ainsi un accès à la chair qui peut être ouvert et refermé si la maturité n'est pas satisfaisante.

16. Les descriptions que Tim donne des arômes des fruits paraissent très sommaires aux clients occidentaux, habitués à la pompe rhétorique qui entoure notamment la consommation de vins.

17. À ces contingences, il faudrait ajouter les pratiques agricoles de Tim, l'âge avancé de ses arbres, en bref tout le collectif qui permet aux durians d'exister comme ils sont, à cet endroit seulement.

18. On mentionnera d'ailleurs que la culture de durians connait une expansion remarquable dans la zone de Johor Baru, frontalière de Singapour (où se situent beaucoup de ceux qui investissent dans le développement de ces plantations). Parce qu'ils n'ont pas toujours les moyens et le temps d'aller vers les fruits, les Singapouriens les font venir à eux.

19. En Europe, les durians que l'on trouve dans les épiceries asiatiques sont essentiellement produits en Thaillande.

\section{RÉSUMÉS}

À travers l'entrée privilégiée du "numb taste ", un goût qui engourdit les muqueuses de la bouche (et qui est ici provoqué par un fruit : le durian), on aborde la question des rapports humains aux aliments de façon transversale, en liant modes de connaissance, de consommation et de production.

Se gardant de toute inflation théorique, le propos s'attache à l'analyse ethnographique méticuleuse du goût et de l'économie générale dans lequel il s'inscrit, entre la Malaisie et Singapour, pour finalement conceptualiser différentes manières de composer et d'éprouver les aliments et les mondes.

On liera notamment l'apparition du numb taste à une contrainte agronomique et ontologique que s'imposent les producteurs et les consommateurs de ces deux pays, en laissant tomber des fruits que la rationalité économique la plus évidente leur enjoint pourtant de récolter.

\section{INDEX}

Mots-clés : goût, numb taste, affects, techniques, cultures végétales, temporalités, durian Index géographique : Malaisie

\section{AUTEUR}

\section{LEO MARIANI}

ATER Laboratoire d'Écoanthropologie et d'Ethnobiologie (UMR 7206) Muséum national d'Histoire naturelle, Paris - leo.mariani@mnhn.fr 